\title{
Resistencia antimicrobiana en Escherichia coli aislada de urocultivos
}

\author{
Antimicrobial resistance in Escherichia coli isolated from urine cultures
}

Resistência antimicrobiana em Escherichia coli isolada de culturas de urina

\author{
Tania Alexandra Panamá Illescas \\ relabse88@gmail.com \\ https://orcid.org/0000-0002-9226-9968
}

\author{
Juan Diego Gallegos Merchan \\ juandye@hotmaill.com \\ https://orcid.org/0000-0001-6764-2911
}

\author{
Neolab Laboratorio Clínico, Ecuador \\ Programa de Maestría en Diagnóstico de Laboratorio Clínico y Molecular, \\ Universidad Católica De Cuenca, Cuenca-Ecuador
}

Recibido 28 de mayo 2021 / Arbitrado y aceptado 30 de julio 2021 / Publicado 14 de diciembre 2021

\begin{abstract}
RESUMEN
Las infecciones del tracto urinario (ITU) son un problema de salud común, la morbilidad por infecciones del tracto urinario adquiridas en la comunidad es alta y el uropatógeno más frecuente en este estudio es Escherichia coli. Objetivo. Determinar la resistencia de antimicrobianos en E. coli aislada de urocultivos, durante Enero - Julio 2019, en pacientes que asistieron al laboratorio clínico Neolab. Materiales y Métodos. La investigación es de tipo cuantitativa, documental, de corte longitudinal descriptiva. Se desarrolló en la ciudad de Cuenca, con un universo de 936 pacientes atendidos en el laboratorio clínico Neolab de Enero a Julio de 2019 con un muestreo no probabilístico por conveniencia con lo cual se obtuvo una muestra de 330 registros de datos, cuyos urocultivos presentaron E. coli. Resultados. Se observó resistencia del $55,15 \%$ en Amoxicilina, Ácido Nalidíxico 50,91 \% y Trimetoprim Sulfametoxazol 46,67\%, Ciprofloxacino 26,67\%, se evidencia mayor resistencia en mujeres tanto en $\beta$-lactámicos, Quinolonas, Sulfas y Macrólidos, se encontró que el mayor número de pacientes se encuentran dentro del grupo de adultos que representa el $54,4 \%$ y adulto mayor con $25,3 \%$. Conclusiones. La resistencia elevada a los antibióticos estudiados, podrían sugerir un uso empírico de los mismos, la detección de estas cifras representa una señal de alarma. La automedicación facilitada por la venta libre de antimicrobianos empeora el problema, por lo que se requiere control estricto y legislación oportuna.
\end{abstract}

Palabras clave: Farmacoresistencia Microbiana; Escherichia coli; Infecciones urinarias

\begin{abstract}
Urinary tract infections (UTI) are a common health problem, morbidity from community-acquired urinary tract infections is high, and the most common uropathogen in this study is Escherichia coli. Objective. To determine the antimicrobial resistance in E. coli isolated from urine cultures, during January - July 2019, in patients who attended the Neolab clinical laboratory. Materials and Methods. The research is quantitative, documentary, descriptive longitudinal cut. It was developed in the city of Cuenca, with a universe of 936 patients treated in the Neolab clinical laboratory from January to July 2019 with a non-probabilistic convenience sampling with which a sample of 330 data records was obtained, whose urine cultures presented E coli. Results. Resistance of $55.15 \%$ was observed in Amoxicillin, Nalidixic Acid $50.91 \%$ and Trimethoprim Sulfamethoxazole $46.67 \%$, Ciprofloxacin $26.67 \%$, greater resistance is evidenced in women both in $\beta$-lactams, Quinolones, Sulfas and Macrolides It was found that the largest number of patients are within the group of adults that represents $54.4 \%$ and the elderly with $25.3 \%$. Conclusions. The high resistance to the studied antibiotics could suggest an empirical use of them, the detection of these figures represents an alarm signal. Self-medication facilitated by the over-the-counter sale of antimicrobials worsens the problem, requiring strict control and timely legislation.
\end{abstract}

Key words: Microbial drug resistance; Escherichia coli; Urinary infections 


\section{RESUMO}

As infecçōes do trato urinário (IU) são um problema de saúde comum, a morbidade das infecções do trato urinário adquiridas na comunidade é alta e o uropógeno mais frequente neste estudo é a Escherichia coli. Objetivo. Para determinar a resistência antimicrobiana em $\mathrm{E}$. coli isolada de culturas de urina, durante janeiro - julho de 2019, em pacientes que freqüentam o laboratório clínico Neolab. Materiais e métodos. Esta é uma pesquisa longitudinal quantitativa, documental e descritiva. Foi desenvolvido na cidade de Cuenca, com um universo de 936 pacientes atendidos no laboratório clínico do Neolab de janeiro a julho de 2019 com uma amostragem não-probabilística por conveniência, com a qual foi obtida uma amostra de 330 registros de dados, cujas culturas de urina apresentaram $\mathrm{E}$. coli. Resultados. Foi observada uma resistência de 55,15\% em Amoxicilina, Ácido Nalidíxico 50,91\% e Trimethoprim Sulfametoxazol 46,67\%, Ciprofloxacina 26,67\%, maior resistência é evidenciada nas mulheres tanto em $\beta$-lactams, Quinolones, Sulfas e Macrolides, verificou-se que o maior número de pacientes está dentro do grupo adulto representando $54,4 \%$ e adulto mais velho com $25,3 \%$. Conclusōes. A alta resistência aos antibióticos estudados poderia sugerir um uso empírico de antibióticos, e a detecção destes números representa um sinal de alarme. A automedicação facilitada pela venda sem prescrição de antimicrobianos agrava o problema, de modo que é necessário um controle rigoroso e legislação oportuna.

Palavras-chave: Resistência a drogas microbianas; Escherichia coli; Infecções do trato urinário

\section{INTRODUCCIÓN}

Las infecciones del tracto urinario (ITU) son un problema de salud común siendo la principal causa de consulta médica, tanto a servicios de emergencia como de consulta ambulatoria en el mundo. La morbilidad por infecciones del tracto urinario adquiridas en la comunidad es alta, el uropatógeno más frecuente es Escherichia coli existiendo en muchas ocasiones un tratamiento empírico (1).

Aunque el tratamiento antimicrobiano tiene buenos resultados en las ITU, tanto complicadas como no complicadas, algunos individuos desarrollan cistitis recurrente, requiriendo profilaxis a largo plazo. Debido al uso indiscriminado de antimicrobianos surgieron cepas resistentes a los antibióticos con el consiguiente descenso de la efectividad en el tratamiento (2). En la literatura nacional e internacional se han publicado recomendaciones para diagnosticar y tratar las infecciones de vías urinarias no complicadas. Sin embargo, la automedicación en países subdesarrollados donde el expendio de antibióticos se realiza de manera ambulatoria y en muchos de los casos la libre adquisición de estos, condicionan una terapia antimicrobiana correcta (3).

Cabe señalar que, E. coli durante muchos años hasta la actualidad ha estado sometida a la acción de múltiples antimicrobianos como Betalactámicos (con inhibidores o sin ellos), fluoroquinolonas, aminoglucósidos, todos presentes en los esquemas terapéuticos de las ITU (4).

En el estudio internacional de la ARESC, sobre resistencia de antimicrobianos a patógenos implicados en infecciones urinarias no complicadas, E. coli mostró una alta resistencia a la sulfonamida SXT $(29,4 \%)$ y una reducida resistencia a la nitrofurantoína $(1,6$ $\%)$ y a fluoroquinolonas ( $8,1 \%$ ) en nueve países europeos y en Brasil (5).

En España se apreció pacientes con infección del tracto urinario por E. coli aislados en orina. El estudio presentó una resistencia a betalactámicos, quinolonas, fosfonatos (6). En Colombia reportaron que el agente bacteriano más frecuente fue $E$. coli con una alta resistencia a betalactámicos y quinolonas (7). En Perú los pacientes con ITUc por E. Coli, 
presentaban resistencia a las quinolonas, cefalosporinas, y aminoglucósidos (8). En Ecuador en años recientes se reporta como bacteria predominante $E$. coli $(84,5 \%)$, la cual presentó una elevada tasa de resistencia a betalactámicos, quinolonas y aminoglucósidos (9).

Las ITU se consideran un problema de salud, esto debido a factores como la elevada ocurrencia, morbilidad, aumento de la tasa de recurrencia, entre otras, lo que se refleja en la calidad de vida del paciente. En este contexto, la resistencia antimicrobiana juega un rol importante en el tratamiento del paciente, por lo que es gran relevancia mantener datos actualizados sobre la susceptibilidad antimicrobiana de uropatógeno como E. Coli.

El objetivo principal fue identificar la resistencia a antimicrobianos en E. coli aislada de urocultivos, en pacientes que asistieron al laboratorio clínico Neolab Ecuador durante el período Enero-Julio 2019.

\section{MATERIALES Y MÉTODOS}

Se realizó un estudio con enfoque cuantitativo, diseño documental, descriptivo. El estudio se desarrolló en la ciudad de Cuenca en Ecuador, el universo comprendió los registros clínicos de 936 pacientes atendidos en el laboratorio clínico Neolab entre EneroJulio 2019, con un muestreo no probabilístico por conveniencia con lo cual se obtuvo una muestra de 330 registros de datos, cuyos urocultivos presentaron E. coli (patógeno objeto de estudio).

Los datos fueron obtenidos de fuentes secundarias seleccionados en la base de datos del laboratorio clínico Neolab, con criterio personal y orden médica clínica de realización de urocultivo.

Criterios de Inclusión. Registro de aislados clínicos identificados con E. coli, que presenten resistencia al menos a un antimicrobiano; y registros de aislados clínicos con orden de urocultivo, que contengan toda la información sobre: Infección del tracto urinario, susceptibilidad antimicrobiana, edad biológica, sexo biológico.

Para el procesamiento, análisis, resumen y presentación de la información, los datos recolectados fueron ingresados en una ficha de registro secundaria que lleva las siguientes variables: infección del tracto urinario; susceptibilidad antimicrobiana; edad biológica, y sexo biológico.

Para el análisis estadístico se generó una base de datos en el programa IBM SPSS Statistics, versión 20.0. Se realizó estadística descriptiva, análisis de frecuencia, prueba de chi-cuadrado, prueba $z$ de comparación de proporciones. Para la presentación de los resultados se utilizaron tablas de simple y doble entrada, tablas cruzadas y los gráficos se representaron mediante diagramas de sectores.

\section{Aspectos éticos}

La investigación que se llevó a cabo se fundamentó en los principios éticos establecidos en la Declaración de Helsinki Adendum de Taiwán 2016. Se dará protección y privacidad a los datos de los pacientes de los cuales se obtuvieron los aislados bacterianos objetos de estudio serán manejados con estricta 
confidencialidad. No se vulnerará el derecho de ningún paciente ya que se resguardará en la base de datos; los nombres de pacientes fueron codificados en clave. La información no se utilizará con otros fines que no sean de carácter investigativo, no tendrán manejo público, no se harán nuevas investigaciones y respetando para lo que fueron tomadas con fines de diagnóstico clínico.

\section{RESULTADOS}

La muestra fue de 330 aislados de urocultivos positivos para E. coli los cuales cumplieron con los criterios de inclusión establecidos para este estudio.
De esta muestra, 317 corresponden a pacientes de sexo femenino que representa el $96,1 \%, 13$ pacientes son de sexo masculino que corresponde a 3,9\% de la muestra. Con relación al total de la población se evidencia una relación de 24:1 con respecto de las mujeres estudiadas a hombres.

En cuanto al grupo etario, el 58,8 \% ( $N=194)$ corresponde a pacientes que se encuentran dentro el grupo de adultos (40-64 años), 75 pacientes $(22,7 \%)$ se encuentran dentro del grupo de adultos mayores ( $>65$ años), 45 niños que representan el $13,6 \%$ y por último 16 adolescentes (4,8 \%) (Tabla 1 ).

\section{Resistencia a antimicrobianos}

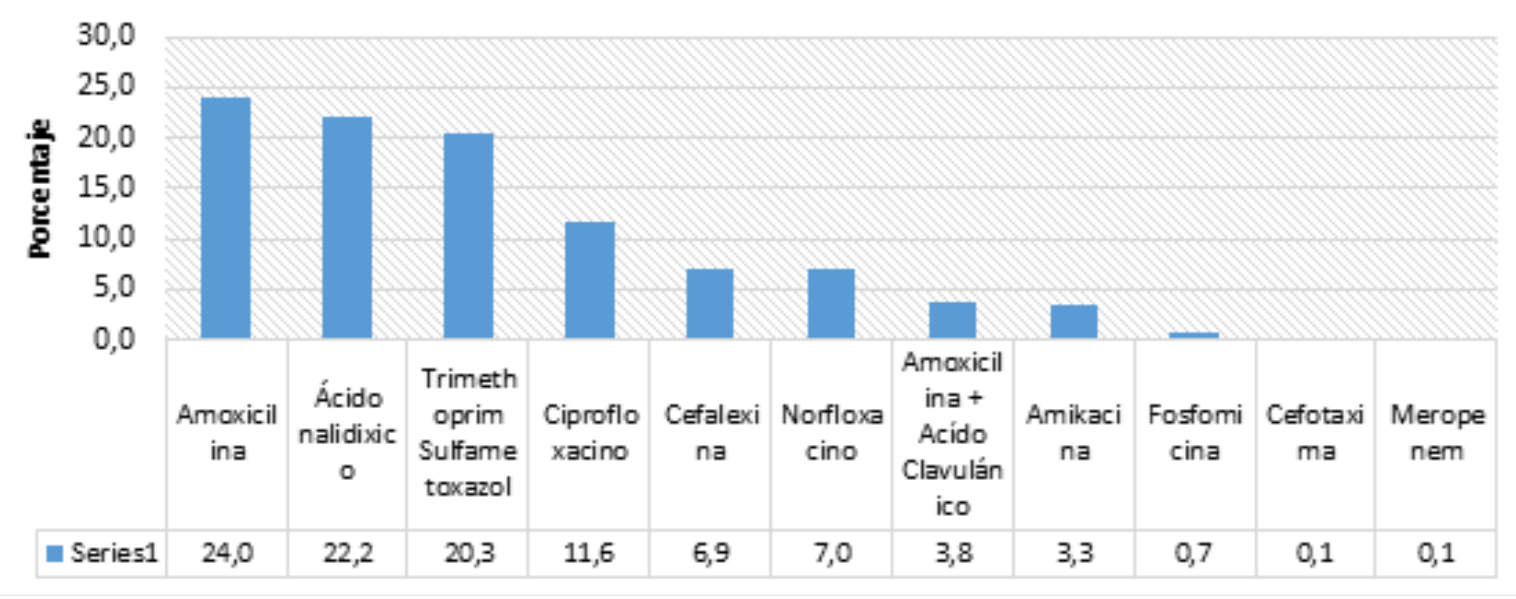

Gráfico 1. Indica los niveles de resistencia de antimicrobianos.

En el Gráfico 1, se observa el porcentaje más elevado de resistencia bacteriana que se presenta en las aminopenicilinas (Amoxicilina), seguido de las Quinolonas de primera generación (Ácido Nalidíxico) y las Sulfas
(Trimetoprim Sulfametoxazol), presentándose en menor porcentaje de resistencia en Cefalosporina de 3ra generación (Cefotaxima) y en Carbapenémicos (Meropenem). 
Tabla 1. Resistencia de E. coli a antibióticos según sexo biológico.

\begin{tabular}{cccc}
\hline & \multicolumn{2}{c}{ Resistencia ATBs } & \\
ATBs & Sexo biológico & \\
& $\mathbf{n}(\%)$ & Masculino & Total \\
& $175(96,2)$ & $\mathbf{n}(\%)$ & \\
\hline Amoxicilina & $7(3,8)$ & 182 \\
Ácido nalidixico & $160(95,2)$ & $8(4,8)$ & 168 \\
Trimethoprim Sulfametoxazol & $145(94,2)$ & $9(5,8)$ & 154 \\
Ciprofloxacino & $81(92,0)$ & $7(8,0)$ & 88 \\
Cefalexina & $48(92,3)$ & $4(7,7)$ & 52 \\
Norfloxacino & $47(88,7)$ & $6(11,3)$ & 53 \\
Amoxicilina + Ácido Clavulánico & $28(96,6)$ & $1(3,4)$ & 29 \\
Amikacina & $25(100)$ & $0(0)$ & 25 \\
Fosfomicina & $5(100)$ & $0(0)$ & 5 \\
Cefotaxima & $1(100)$ & $0(0)$ & 1 \\
Meropenem & $1(100)$ & $0(0)$ & 1 \\
\hline
\end{tabular}

Leyenda: ATBs (antimicrobianos)

En este estudio se puede evidenciar el total de pacientes que presentan E. coli con resistencia a 1 o más antibióticos de los 11 testeados para el estudio. En donde se aprecia la relación entre la resistencia antibacteriana y el sexo biológico, en la cual se evidencia mayor frecuencia de resistencia en mujeres tanto en $\beta$-lactámicos, Quinolonas, Sulfas y Macrólidos. Sin embargo, resulta escasa la resistencia a fosfonatos y carbapenémicos (Tabla 2).

Tabla 2. Análisis de grados de susceptibilidad a antimicrobianos determinados como resistentes, intermedio y sensible a cada fármaco en relación con el sexo biológico.

\begin{tabular}{cccccccc}
\hline & Sensibilidad & \multicolumn{3}{c}{ Sexo biológico } & \multicolumn{3}{c}{ Total } \\
Antimicrobianos & \multicolumn{2}{c}{ Femenino } & \multicolumn{2}{c}{ Masculino } & \multicolumn{2}{c}{} \\
& antimicrobiana & $\mathbf{n}$ & $\%$ & $\mathbf{n}$ & $\%$ & $\mathbf{n}$ & $\%$ \\
\hline \multirow{3}{*}{ Ciprofloxacino } & Resistente & 81 & 24,5 & 7 & 2,1 & 88 & 26,7 \\
& Intermedio & 2 & 0,6 & 0 & 0,0 & 2 & 0,6 \\
& Sensible & 234 & 70,9 & 6 & 1,8 & 240 & 72,7 \\
\hline
\end{tabular}




\begin{tabular}{|c|c|c|c|c|c|c|c|}
\hline \multirow{3}{*}{ Antimicrobianos } & \multirow{3}{*}{$\begin{array}{c}\text { Sensibilidad } \\
\text { antimicrobiana }\end{array}$} & \multicolumn{4}{|c|}{ Sexo biológico } & \multirow{2}{*}{\multicolumn{2}{|c|}{ Total }} \\
\hline & & \multicolumn{2}{|c|}{ Femenino } & \multicolumn{2}{|c|}{ Masculino } & & \\
\hline & & $n$ & $\%$ & $\mathbf{n}$ & $\%$ & $\mathrm{n}$ & $\%$ \\
\hline \multirow{3}{*}{ Norfloxacino } & Resistente & 47 & 14,2 & 6 & 1,8 & 53 & 16,1 \\
\hline & Intermedio & 2 & 0,6 & 0 & 0,0 & 2 & 0,6 \\
\hline & Sensible & 268 & 81,2 & 7 & 2,1 & 275 & 83,3 \\
\hline \multirow{3}{*}{ Ácido nalidíxico } & Resistente & 160 & 48,5 & 8 & 2,4 & 168 & 50,9 \\
\hline & Intermedio & 2 & 0,6 & 0 & 0,0 & 2 & 0,6 \\
\hline & Sensible & 155 & 47,0 & 5 & 1,5 & 160 & 48,5 \\
\hline \multirow{3}{*}{ Cefalexina } & Resistente & 48 & 14,5 & 4 & 1,2 & 52 & 15,8 \\
\hline & Intermedio & 4 & 1,2 & 0 & 0,0 & 4 & 1,2 \\
\hline & Sensible & 265 & 80,3 & 9 & 2,7 & 274 & 83,0 \\
\hline \multirow{3}{*}{ Cefotaxima } & Resistente & 1 & 0,3 & 0 & 0,0 & 1 & 0,3 \\
\hline & Intermedio & 1 & 0,3 & 0 & 0,0 & 1 & 0,3 \\
\hline & Sensible & 315 & 95,5 & 13 & 3,9 & 328 & 99,4 \\
\hline \multirow{2}{*}{ Meropenem } & Resistente & 1 & 0,3 & 0 & 0,0 & 1 & 0,3 \\
\hline & Sensible & 316 & 95,8 & 13 & 3,9 & 329 & 99,7 \\
\hline \multirow{2}{*}{ Amikacina } & Resistente & 25 & 7,6 & 0 & 0,0 & 25 & 7,6 \\
\hline & Sensible & 292 & 88,5 & 13 & 3,9 & 305 & 92,4 \\
\hline \multirow{3}{*}{ Fosfomicina } & Resistente & 5 & 1,5 & 0 & 0,0 & 5 & 1,5 \\
\hline & Intermedio & 1 & 0,3 & 0 & 0,0 & 1 & 0,3 \\
\hline & Sensible & 311 & 94,2 & 13 & 3,9 & 324 & 98,2 \\
\hline \multirow{3}{*}{ Amoxicilina } & Resistente & 175 & 53,0 & 7 & 2,1 & 182 & 55,2 \\
\hline & Intermedio & 3 & 0,9 & 0 & 0,0 & 3 & 0,9 \\
\hline & Sensible & 139 & 42,1 & 6 & 1,8 & 145 & 43,9 \\
\hline \multirow{3}{*}{$\begin{array}{c}\text { Amoxicilina + } \\
\text { Ácido } \\
\text { Clavulánico }\end{array}$} & Resistente & 28 & 8,5 & 1 & 0,3 & 29 & 8,8 \\
\hline & Intermedio & 29 & 8,8 & 2 & 0,6 & 31 & 9,4 \\
\hline & Sensible & 260 & 78,8 & 10 & 3,0 & 270 & 81,8 \\
\hline \multirow{3}{*}{$\begin{array}{l}\text { Trimethoprim } \\
\text { Sulfametoxazol }\end{array}$} & Resistente & 145 & 43,9 & 9 & 2,7 & 154 & 46,7 \\
\hline & Intermedio & 1 & 0,3 & 0 & 0,0 & 1 & 0,3 \\
\hline & Sensible & 171 & 51,8 & 4 & 1,2 & 175 & 53,0 \\
\hline
\end{tabular}


Al realizar el análisis de grados de susceptibilidad a antimicrobianos determinados como resistentes, intermedio y sensible a cada fármaco con relación al sexo biológico, se encontró mayor grado de resistencia a las aminopenicilinas, seguido de quinolonas de primera generación y sulfas, y con relación al menor porcentaje en cefalosporinas y Carbapenémicos estudiados.
Con respecto a la susceptibilidad bacteriana por grupos etarios, se observa que el grupo de adultos presenta mayor población con resistencia bacteriana a todos los antimicrobianos estudiados, en la Tabla 3 se expone los resultados.

Tabla 3. Resistencia a Antimicrobianos según grupo Etario.

\begin{tabular}{|c|c|c|c|c|c|c|c|}
\hline \multirow[b]{2}{*}{ Antimicrobianos } & \multirow{2}{*}{$\begin{array}{c}\text { Sensibilidad } \\
\text { antimicrobiana }\end{array}$} & & \multicolumn{4}{|c|}{ Grupos Etarios } & \multirow[b]{2}{*}{ Total } \\
\hline & & & Niñez & Adolescencia & Adultos & $\begin{array}{l}\text { Adulto } \\
\text { mayor }\end{array}$ & \\
\hline \multirow{2}{*}{ Ciprofloxacino } & \multirow[t]{2}{*}{ Resistente } & $\mathrm{n}$ & 8 & 4 & 52 & 24 & 88 \\
\hline & & $\%$ & $9,1 \%$ & $4,5 \%$ & $59,1 \%$ & $27,3 \%$ & $100,0 \%$ \\
\hline \multirow{2}{*}{ Norfloxacino } & \multirow[t]{2}{*}{ Resistente } & $\mathrm{n}$ & 5 & 2 & 30 & 16 & 53 \\
\hline & & $\%$ & $9,4 \%$ & $3,8 \%$ & $56,6 \%$ & $30,2 \%$ & $100,0 \%$ \\
\hline \multirow{2}{*}{ Ácido nalidíxico } & \multirow[t]{2}{*}{ Resistente } & $\mathrm{n}$ & 19 & 8 & 97 & 44 & 168 \\
\hline & & $\%$ & $11,3 \%$ & $4,8 \%$ & $57,7 \%$ & $26,2 \%$ & $100,0 \%$ \\
\hline \multirow{2}{*}{ Cefalexina } & \multirow[t]{2}{*}{ Resistente } & $\mathrm{n}$ & 11 & 3 & 24 & 14 & 52 \\
\hline & & $\%$ & $21,2 \%$ & $5,8 \%$ & $46,2 \%$ & $26,9 \%$ & $100,0 \%$ \\
\hline \multirow{2}{*}{ Cefotaxima } & \multirow[t]{2}{*}{ Resistente } & $\mathrm{n}$ & 0 & 0 & 1 & 0 & 1 \\
\hline & & $\%$ & $0,0 \%$ & $0,0 \%$ & $100,0 \%$ & $0,0 \%$ & $100,0 \%$ \\
\hline \multirow{2}{*}{ Meropenem } & \multirow[t]{2}{*}{ Resistente } & $\mathrm{n}$ & 0 & 0 & 1 & 0 & 1 \\
\hline & & $\%$ & $0,0 \%$ & $0,0 \%$ & $100,0 \%$ & $0,0 \%$ & $100,0 \%$ \\
\hline \multirow{2}{*}{ Amikacina } & \multirow[t]{2}{*}{ Resistente } & $\mathrm{n}$ & 3 & 2 & 14 & 6 & 25 \\
\hline & & $\%$ & $12,0 \%$ & $8,0 \%$ & $56,0 \%$ & $24,0 \%$ & $100,0 \%$ \\
\hline \multirow{2}{*}{ Fosfomicina } & \multirow[t]{2}{*}{ Resistente } & $n$ & 3 & 0 & 1 & 1 & 5 \\
\hline & & $\%$ & $60,0 \%$ & $0,0 \%$ & $20,0 \%$ & $20,0 \%$ & $100,0 \%$ \\
\hline \multirow{2}{*}{ Amoxicilina } & \multirow[t]{2}{*}{ Resistente } & $\mathrm{n}$ & 27 & 10 & 99 & 46 & 182 \\
\hline & & $\%$ & $14,8 \%$ & $5,5 \%$ & $54,4 \%$ & $25,3 \%$ & $100,0 \%$ \\
\hline \multirow{2}{*}{$\begin{array}{l}\text { Amoxicilina + } \\
\text { Ácido Clavulánico }\end{array}$} & \multirow[t]{2}{*}{ Resistente } & $\mathrm{n}$ & 7 & 2 & 14 & 6 & 29 \\
\hline & & $\%$ & $24,1 \%$ & $6,9 \%$ & $48,3 \%$ & $20,7 \%$ & $100,0 \%$ \\
\hline \multirow{2}{*}{$\begin{array}{c}\text { Trimetoprim } \\
\text { Sulfametoxazol }\end{array}$} & \multirow[t]{2}{*}{ Resistente } & $\mathrm{n}$ & 21 & 9 & 85 & 39 & 154 \\
\hline & & $\%$ & $13,6 \%$ & $5,8 \%$ & $55,2 \%$ & $25,3 \%$ & $100,0 \%$ \\
\hline
\end{tabular}


Al estudiar la resistencia según los grupos etarios, se encontró que Amoxicilina es el antibiótico con más alto porcentaje de resistencia. El mayor número de pacientes resistentes se encuentran dentro del grupo de adultos que representa el 54,4\% ( $\mathrm{N}=99)$, a este grupo le sigue el de adulto mayor con 46 pacientes (25,3\%), es importante destacar que existen 27 muestras que pertenecen $(14,8 \%)$ al grupo etario considerados como niños.

En relación Ácido nalidíxico que dentro de las estadísticas es el segundo que presenta mayor resistencia, el grupo de adultos es el que presenta mayor resistencia ( $97 / 57,7 \%)$, el segundo grupo es el del adulto mayor (44/26,2 $\%)$.

\section{DISCUSIÓN}

Las infecciones del tracto urinario (ITU) son un problema de salud común que, con mayor frecuencia se presenta en mujeres, siendo E. coli el microorganismo identificado como agente causal más común. Aunque el tratamiento antimicrobiano tiene buenos resultados en las ITU tanto complicadas como no complicadas, algunos individuos desarrollan cistitis recurrente, requiriendo profilaxis a largo plazo. Debido al uso indiscriminado de antimicrobianos surgieron cepas resistentes a los antibióticos con el consiguiente descenso de la efectividad en el tratamiento; la automedicación en países subdesarrollados donde el expendio de antibióticos se realiza de manera ambulatoria y en muchos de los casos la libre adquisición de estos condiciona una terapia antimicrobiana correcta.

En la investigación mencionada anteriormente, se puede apreciar que, de acuerdo con las tendencias globales, los resultados revelan mayor prevalencia de infecciones del tracto urinario en mujeres que en los hombres. Además, las tasas de resistencia a los antimicrobianos fueron superiores en pacientes femeninas que en varones. lo que concuerda con la investigación de Linhares y col., que señalan que las ITU fueron más frecuentes en mujeres $(78,5 \%)$ y su incidencia varió con la edad, afectando más a los pacientes ancianos (38,6\%) (10) en relación a este último dato, esta investigación encontró que el mayor número de pacientes se encuentran dentro del grupo de adultos que representa el 54,4\%, a este grupo le sigue el de adulto mayor $25,3 \%$.

En relación con el total de pacientes que presentan $E$. coli con resistencia a 11 antimicrobianos, se observó que el porcentaje más elevado de resistencia bacteriana se presenta en las aminopenicilinas (Amoxicilina), seguido de las Quinolonas de primera generación (Ácido Nalidíxico) y las Sulfas (Trimetoprim Sulfametoxazol), presentándose en menor porcentaje de resistencia en Cefalosporina de 3ra generación (Cefotaxima) y en Carbapenémicos (Meropenem). Aunque el diagnóstico delas ITU sebasa en la confirmación microbiológica, está ampliamente aceptada 
la recomendación de iniciar un tratamiento antibiótico empírico precoz en la mayor parte de los casos sospechosos, con vistas a tratar de evitar las potenciales complicaciones derivadas del cuadro infeccioso (11) lo que podría estar relacionado en los hallazgos de esta investigación.

Al revisar literatura relacionada, se encontró el estudio realizado en Francia en 2016 por Lavigne et al., que muestra niveles de resistencia similares a este estudio, así, indican que la resistencia de aislados de $E$. coli a amoxicilina, ticarcilina, ácido nalidíxico presentan cifras del $61,4 \%, 59 \%$ y $31,9 \%$, respectivamente(12). Los autores de esta investigación concluyen que es importante el establecimiento de directrices para la elección de antibióticos de primera línea criterio con el cual se coincide.

De igual manera, Erb et al., realizaron pruebas de susceptibilidad a los antimicrobianos para 5246 cepas de E. coli en orina, encontrando que la resistente a amoxicilina fue de $43,1 \%$, cotrimoxazol $24,5 \%$ y ciprofloxacino $17,4 \%$. Las tasas de resistencia fueronbajas parameropenem $0,0 \%$, fosfomicina $0,9 \%$ y nitrofurantoína $1,5 \%$. Valores similares a los hallados en esta investigación en relación a fosfomicina y meropenem (13).

En los estudios consulados de algunos países, se pudo observar una resistencia creciente al Trimetoprim sulfametoxazol, que se usa ampliamente como antimicrobiano de primera línea en el tratamiento de infecciones urinarias no complicadas. Así, Bartoletti et al., 2016 indica que la resistencia a TMPSMZ se encuentra por encima del nivel del $20 \%$, indicando que TMP-SMZ no debería ser utilizado en tratamientos empíricos(14). Una alta tasa resistencia a TMP-SMZ (24,5\%) en E. coli aislado de orina en 2012-2015 fue informado en Suiza por Erb et al. (14).

El uso generalizado de Fluoroquinolonas $(\mathrm{FQ})$, especialmente ciprofloxacino, en pacientes ambulatorios es la causa de un continuo aumento de la resistencia a estos fármacos. La resistencia a las FQ es significativamente mayor en los países en desarrollo (55,5\% a $85,5 \%$ ) que en países desarrollados (5,1-32,0\%) (15).

En referencia a ciprofloxacino, este estudio arrojo una resistencia del $26,67 \%$, no esperado en relación con los reportes a nivel internacional que se tienen.

La resistencia a Fluoroquinolonas (FQ) se ha reportado ampliamente a nivel mundial, Fasugba et al., en 2015 indicaron que los resultados del metaanálisis realizado sobre la resistencia a ciprofloxacino en las infecciones urinarias adquiridas en la comunidad y en el hospital mostraron que la resistencia de E. coli en infecciones urinarias al ciprofloxacino era mayor en el hospital en comparación con el entorno comunitario (16).

Así mismo, estudios realizados por Prasada y col., (2019) en India reveló una alta tasa de resistencia de fluoroquinolonas ( $>60 \%)$. En Polonia, se observó resistencia a las FQ en 
alrededor del 30\% de UPEC (17) Walker et al., en 2016 demostraron tasas mucho más altas de resistencia a las $\mathrm{FQ}$, que van del $49 \%$ al $72 \%$ (18).

Una mayor tasa de resistencia a ciprofloxacino se detectó en pacientes ancianos hospitalizados en Argentina (42,8\%) (19) y en infecciones aislados de la comunidad e infecciones hospitalarias en México (47,3\%) (20).

Moreira y col en estudios realizados en pacientes ambulatorios en Brasilia, encontraron el $18,8 \%$ de resistencia a ciprofloxacino, y la misma que se asoció con resistencia a múltiples fármacos (21).

Luego de revisar los resultados citados anteriormente y que difieren con la presente investigación, se debería ampliar la investigación para contrastar este hallazgo.

En lo que se refiere a las resistencias de cefalexina (beta), norfloxacino (quinolonas) amoxicilina + ácido clavulánico, dentro de este estudio encontramos resistencias en el orden del $15,76 \%, 16,06 \%, 8,79 \%$ respectivamente.

En comparación a esta investigación, encontramos que en el estudio realizado por Moya y col., indican que encontraron una resistencia del $49 \%$ a cefalosporinas de primera generación, también informan que en el $86 \%$ de los casos fueron sensibles a amoxicilina/ clavulánico (11) datos que difieren de los encontrados en este análisis, por lo que como se sugirió anteriormente se debería ampliar la investigación.
En Ecuador, estudios realizados por Rodríguez en 2013, indican que se encontró una resistencia bacteriana al Trimetoprim sulfametoxazol en $63 \%$, seguido de Ciprofloxacino con 39\% y Amoxicilina/ Ácido clavulánico con 29\% (22). Gordillo y col., en un estudio realizado en Quito en 2018 encontraron en su investigación que Ácido nalidíxico presenta una resistencia del 74,3 $\%$, Ciprofloxacino 56,8 \% y Trimetoprim sulfametoxazol $57,6 \%$ (23) estos resultados concuerdan con nuestros análisis a excepción de ciprofloxacino ya que en este estudio obtuvimos un porcentaje menor.

La literatura indica que la prevalencia de la resistencia a los antibióticos está empeorando, probablemente debido al número creciente de cepas bacterianas multirresistentes y al uso indiscriminado de antibióticos de amplio espectro y tratamiento empírico (14).

Se indica además de tener en cuenta las tasas de resistencia local, se debe considerar los factores de riesgo del paciente frente a la resistencia a antibióticos y a los principios farmacológicos, los mismos que ayudarán a guiar el tratamiento empírico óptimo de las infecciones urinarias (24).

\section{CONCLUSIONES}

Se observó una elevada resistencia a las aminopenicilinas, Quinolonas y Sulfas, también que existe una alta resistencia en pacientes adultos, así como en pacientes de 
sexo femenino, por lo cual la detección de estas cifras representa una señal de alarma que motiva una continua vigilancia de la resistencia a los antimicrobianos en pacientes adultos con infecciones urinarias producidas por E. coli

Se recomienda que la elección de la terapia antibiótica para tratar ITU, en la medida de lo posible, esta sea observada mediante el urocultivo y el antibiograma.

\section{REFERENCIAS BIBLIOGRÁFICAS}

1. Guajardo C, González P, Ayala J Resistencia antimicrobiana en la infección urinaria por Escherichia coli adquirida en la comunidad: ¿Cuál antibiótico voy a usar? Salud Pública de México.2009;51(2):155-9. Disponible en: http:// www.scielo.org.mx/scielo.php?script $=$ sci_ abstract\&pid=S0036-36342009000200012\&lng =es\&nrm=iso\&tlng=es

2. Chávez V. Patrones de resistencia antimicrobiana y etiología en infecciones urinarias no complicadas.2010;146(4):5. Disponible en: https://www.medigraphic.com/ cgi-bin/new/resumen.cgi?IDARTICULO=26783

3. Sánchez J, Guillán C, Fuster C, Madrid F, Jiménez M, García J. Sensibilidad microbiana de escherichia coli en infecciones urinarias extrahospitalarias. Actas Urológicas Españolas.2003;27(10):783-7. Disponible en: http://scielo.isciii.es/scielo.php?script=sci_ abstract\&pid=S0210-48062003001000003\&lng =es\&nrm=iso\&tlng=es

4. Trueba B, Samper $Y$, Espinosa F, Casares M, Rodríguez N, María D, et al. Susceptibilidad antimicrobiana y mecanismos de resistencia de Escherichia coli aisladas a partir de urocultivos en un hospital de tercer nivel. Revista Cubana de Medicina.:11. Disponible en: https://www. medigraphic.com/cgi-bin/new/resumen. cgi?IDARTICULO=51171

5. Schito C, Naber G, Botto H, Palou J, Mazzei T, Gualco L,et al.TheARESCstudy:aninternational survey on the antimicrobial resistance of pathogens involved in uncomplicated urinary tract infections. International Journal of Antimicrobial Agents.2009;34(5):407-13. Disponible en: https://www.sciencedirect.com/ science/article/pii/S0924857909002143

6. Losada I, Barbeito G, García F, Fernández B, Malvar A, Hervada X, et al. Estudio de sensibilidad de Escherichia coli productores de infecciones del tracto urinario comunitarias en Galicia. Período: 2016-2017. Atención Primaria.2020;52(7):462-8. Disponible en: https://www.sciencedirect.com/science/ article/pii/S0212656718304748

7. Castro R, Barreto $A C$, Guzmán H, Ortega $\mathrm{R}$, Benítez $\mathrm{L}$. Patrones de resistencia antimicrobiana en uropatógenos gramnegativos aislados de pacientes ambulatorios y hospitalizados Cartagena, 2005-2008. Rev salud pública.2010;12:1010-9. Disponible en: https://scielosp.org/article/ rsap/2010.v12n6/1010-1019/

8. Montañez $R$, Montenegro J, Arenas $F$, Vásquez R. Infección urinaria alta comunitaria por E. coli resistente a ciprofloxacino: características asociadas en pacientes de un hospital nacional en Perú. Anales de la Facultad de Medicina.2015;76(4):385-91. Disponible en: http://www.scielo.org.pe/ scielo.php?script=sci_abstract\&pid=S1025$55832015000500009 \& \operatorname{lng}=$ es\&nrm=iso\&tlng= es

9. Chávez A, Castillo R, Alcocer A, Flores $Y$, Puga E, Buitrón E. "Resistencia y sensibilidad bacteriana en urocultivos en una población de mujeres de Ecuador". Revista Med de la 
Facultad de Medicina.2018;26(2):6. Disponible en: https://dialnet.unirioja.es/servlet/ articulo?codigo $=7311175$

10. Linhares I, Raposo $T$, Rodrigues A, Almeida A. Frequency and antimicrobial resistance patterns of bacteria implicated in community urinary tract infections: a ten-year surveillance study (2000-2009). BMC Infect Dis.2013;13:19. Disponible en: https://www.ncbi.nlm.nih.gov/ pmc/articles/PMC3556060/

11. Moya V, Díaz M, Ibáñez A, Suárez P, Martínez $V$, Ordóñez $F$, et al. Patrón de aislamiento bacteriano y sensibilidad antimicrobiana en urocultivos positivos obtenidos de una población pediátrica. Revista Española de Quimioterapia.2016;29(3):146-50. Disponible en: https://medes.com/publication/110538

12. Levigne A. Resistance and virulence potential of uropathogenic Escherichia coli strains isolated from patients hospitalized in urology departments: a French prospective multicentre study | Microbiology Society. Disponible en: https://pubmed.ncbi.nlm.nih. gov/26953145/

13. Erb $S$, Frei R, Tschudin S, Egli A, Dangel $M$, Bonkat $G$, et al. Basic patient characteristics predict antimicrobial resistance in $\mathrm{E}$. coli from urinary tract specimens: a retrospective cohort analysis of 5246 urine samples. Swiss Medical Weekly.2018;148(4546). Disponible en: https:// smw.ch/article/doi/smw.2018.14660

14. Bartoletti R, Cai T, Wagenlehner F, Naber $\mathrm{K}$, Bjerklund T. Treatment of Urinary Tract Infectionsand AntibioticStewardship. European Urology Supplements.2016;15(4):81-7. Disponible en: https://www.sciencedirect.com/ science/article/pii/S1569905616300495

15. KOT B. Antibiotic Resistance Among Uropathogenic Escherichia coli. Pol J Microbiol.2019;68(4):403-15. Disponible en: https://www.ncbi.nlm.nih.gov/pmc/articles/ PMC7260639/
16. Fasugba $O$, Gardner A, Mitchell $B$, Mnatzaganian G. Ciprofloxacin resistance in community- and hospital-acquired Escherichia coli urinary tract infections: a systematic review and meta-analysis of observational studies. BMC Infect Dis.2015;15. Disponible en: https://www.ncbi.nlm.nih.gov/pmc/articles/ PMC4660780/

17. Michno $M$, Sydor A, Wałaszek $M$, Sułowicz W. Microbiology and Drug Resistance of Pathogens in Patients Hospitalized at the Nephrology Department in the South of Poland. Pol J Microbiol.2018;67(4):517-24. Disponible en: https://pubmed.ncbi.nlm.nih. gov/30550238/

18. Walker E, Lyman A, Gupta K, Mahoney V, Snyder M, Hirsch B. Clinical Management of an Increasing Threat: Outpatient Urinary Tract Infections Due to Multidrug-Resistant Uropathogens.Clin Infect Dis.2016;63(7):9605. Disponible en: https://pubmed.ncbi.nlm.nih. gov/27313263/

19. Delpech G, Allende G, Lissarrague S, Sparo $M$. Antimicrobial Resistance of Uropathogenic Escherichia coli from Elderly Patients at a General Hospital, Argentina. The Open Infectious Diseases Journal.2018;10(1). Disponible en: https://benthamopen.com/ FULLTEXT/TOIDJ-10-79

20. Ramírez F, Moreno A, Avelar F, Márquez F, Harel J, Guerrero A. An evaluation of multidrug-resistant Escherichia coli isolates in urinary tract infections from Aguascalientes, Mexico: cross-sectional study. 2018;17(1):34. Disponible en: https://www.ncbi.nlm.nih.gov/ pmc/articles/PMC6057003/

21. Moreira C, Martins J, Gonçalves F, Martins V, Melo B, Pitondo A, et al. Ciprofloxacin resistance in uropathogenic Escherichia coli isolates causing community-acquired urinary infections in Brasília, Brazil. Journal of Global Antimicrobial Resistance.2017;9:61-7. 
Disponible en: https://www.sciencedirect.com/ science/article/pii/S2213716517300450

22. Rodríguez A, Torres C. "Resistencia Bacteriana en Urocultivos, en Pacientes del Área de Hospitalización del Hospital Un Canto a la Vida «Padre Carolo», en el Período comprendido entre Enero 2012 a Diciembre 2013". 2016:83. Disponible en: http://www. dspace.uce.edu.ec/handle/25000/7773

23. Gordillo F, Barrera F. Perfil de resistencia de uropatógenos en pacientes con diabetes en Quito, Ecuador, inquietante panorama. Salud Publica Mex.2017;60(1, ene-feb):97. Disponible en: http://saludpublica.mx/index.php/spm/ article/view/8756
24. Walker E, Lyman A, Gupta K, Mahoney V, Snyder M, Hirsch B. Clinical Management of an Increasing Threat: Outpatient Urinary Tract Infections Due to Multidrug-Resistant Uropathogens. Clin Infect Dis.2016;63(7):9605. Disponible en: https://pubmed.ncbi.nlm.nih. gov/27313263/

Conflicto de intereses: Ninguno declarado por los autores.

Financiación: Ninguna declarada por los autores.

Agradecimiento: Ninguno manifestado por los autores

\section{ACERCA DE LOS AUTORES}

Tania Alexandra Panamá Illescas. Química Farmaceuta. Jefa de laboratorio, Laboratorio clínico Relabse, Cuenca. Universidad Católica de Cuenca, Ecuador.

Juan Diego Gallegos Merchán. Doctor en Laboratorio Clínico y Administración de Servicios de Salud. Diplomado En Microbiología Avanzada. Magister en Microbiología Mención Biomédica CN. Facilitador Académico Externo del Ces. Coordinador del Programa Meningitis y Neumonías Bacterianas Agudas. Universidad Católica de Cuenca, Ecuador. 